\title{
Novel Situations of Endothelial Injury in Stroke - Mechanisms of Stroke and Strategy of Drug Development: Intracranial Bleeding Associated With the Treatment of Ischemic Stroke: Thrombolytic Treatment of Ischemia-Affected Endothelial Cells With Tissue-Type Plasminogen Activator
}

\author{
Yasuhiro Suzuki ${ }^{1, *}$, Nobuo Nagai $^{2}$, and Kazuo Umemura ${ }^{1}$ \\ ${ }^{1}$ Department of Pharmacology, Hamamatsu University School of Medicine, Shizuoka 431-3192, Japan \\ ${ }^{2}$ Department of Animal Bioscience, Faculty of Bioscience, Nagahama Institute of Bio-Science and Technology, \\ Nagahama, Shiga 526-0829, Japan
}

Received October 18, 2010; Accepted December 20, 2010

\begin{abstract}
Previous studies have shown that the risk of intracranial hemorrhage (ICH) associated with the treatment of ischemic stroke is mainly attributable to antithrombotic agents. On the basis of clinical trials, only tissue-type plasminogen activator (t-PA) has been approved for treating acute ischemic strokes, but delayed treatment with t-PA is associated with the risk of cerebral hemorrhagic transformation of ischemic stroke. t-PA converts plasminogen to plasmin, which participates primarily in clot lysis via fibrin degradation and, to some extent, in tissue remodeling via degradation of various extracellular matrix proteins, either directly or via activation of matrix metalloproteinases (MMPs). MMPs mediate the pathogenesis of ischemic-stroke-associated ICH by causing the disruption of vasculature. In particular, the binding of t-PA with one of its receptors leads to the activation of low-density lipoprotein receptor-related protein (LRP), which in turn results in the release of MMP-3 by endothelial cells. LRP production is reported to be upregulated in endothelial cells exposed to ischemia, and elevated LRP levels have been implicated in the increased ICH risk associated with delayed t-PA treatment. This implies that the t-PA / LRP / MMP3 pathway may be a suitable target for developing strategies to improve the therapeutic efficacy of $\mathrm{t}$-PA in acute ischemic stroke.
\end{abstract}

Keywords: tissue-type plasminogen activator, cerebral ischemia, intracranial hemorrhage, matrix metalloproteinase, low-density lipoprotein receptor-related protein, endothelial injury

\section{Introduction}

Previous studies have reported that the risk of intracranial hemorrhage (ICH) associated with the treatment of ischemic stroke is mainly attributable to antithrombotic agents. Among these drugs, antiplatelet agents such as aspirin, ticlopidin, and clopitdogrel have been reported to cause $\mathrm{ICH}$ in $0.3 \%-0.4 \%$ of patients treated with these drugs (1); furthermore, the rate of ICH in subjects treated

*Corresponding author. yapplel@hama-med.ac.jp Published online in J-STAGE on April 16, 2011 (in advance) doi: 10.1254/jphs.10R27FM by the oral anticoagulant warfarin has been reported to be 1.2 per 100 patient-years (2). Although primary prevention trials conducted on patients with a history of ischemic stroke have shown that oral anticoagulant therapy reduces the risk of subsequent strokes by $60 \%-70 \%$ (3), $23.3 \%$ of the patients administered heparin as early anticoagulant treatment have been reported to develop symptomatic cerebral hemorrhages $(4,5)$. A previous study reported that compared to aspirin, dalteparin, low-molecular-weight heparin was not superior in its beneficial effects but caused more severe hemorrhages (6). The effects of cilostazol, an antiplatelet drug that inhibits cyclic adenosine monophosphate 
(AMP) phosphodiesterase, on the recurrence of cerebral infarction have been evaluated in the Cilostazol Stroke Prevention (CPSP) -2 study (7). The results of this study showed that the rate of cerebral hemorrhage and subarachnoid hemorrhage was $0.34 \%$ among patients treated with cilostazol (100 $\mathrm{mg}$ twice daily), which was less than that among patients treated with aspirin $(0.96 \%, 81 \mathrm{mg}$ every day).

Thrombolytic therapy by urokinase (u-PA) in patients with cerebral infarction was first attempted in the 1970's (8). u-PA promotes the prompt and sustained increase of plasma thrombolytic activity, with relatively minor disturbance of the blood coagulation system. However, several patients treated with this therapy developed hemorrhagic complications and the therapeutic effects of this therapy were not favorable. The Prolyse in Acute Cerebral Thromboembolism (PROACT) II trial revealed that intra-arterial treatment with pro-uPA plus heparin within $6 \mathrm{~h}$ after stroke onset caused by middle cerebral artery (MCA) occlusion significantly improved the clinical outcome at 90 days, despite an increase in the rate of early symptomatic ICH (9). In the PROACT II trial, ICH with neurological deterioration within $24 \mathrm{~h}$ of the onset of stroke occurred in $10 \%$ of the patients treated with pro-uPA and in $2 \%$ of the patients treated only with heparin. The Middle Cerebral Artery Embolism Local Fibrinolytic Intervention Trial (MELT) Japan was conducted to determine the safety and clinical efficacy of administering an intra-arterial infusion of u-PA within $6 \mathrm{~h}$ of the onset of stroke (10). The rate of ICH occurring within 24 $\mathrm{h}$ of the onset of stroke was $9 \%$ among u-PA-treated patients, including one patient with hematoma caused by guide-wire perforation, whereas it was $2 \%$ in patients not treated with intra-arterial infusion of u-PA. The Independent Monitoring Committee recommended that MELT Japan be discontinued after intravenous infusion of recombinant tissue-type plasminogen activator ( $t-P A)$ was approved in Japan (10). The trial was aborted prematurely, and the primary end point did not reach statistical significance.

Intravenous administration of t-PA within $3 \mathrm{~h}$ of the onset of stroke has been approved for treatment for acute ischemic stroke. However, this therapy is associated with increased risk of potentially life-threatening symptomatic ICH. Robust data on the rate of ICH among patients treated with intravenously administered t-PA has been collected from several large randomized trials (11) and observational stroke registries (12). These data indicate that the risk of symptomatic ICH in patients treated with intravenously administered thrombolytic agents ranges between $2 \%$ and $8 \%$. In the European Cooperative Acute Stroke Study III (ECASS III) (13), t-PA was administered $3.0-4.5 \mathrm{~h}$ after the onset of stroke; the results of this study showed that t-PA treatment in this manner afforded significantly better outcomes in the primary endpoint than placebo administration as well as treatment within the $3 \mathrm{~h}$ window period, without any difference in the mortality rates between the treatment groups. Furthermore, there was no significant difference in the risk of symptomatic ICH among patients administered t-PA within the $0-3 \mathrm{~h}$ window period and that among patients administered t-PA during the $3.0-4.5$ window period; however, the rate of ICH among the former was lower than that among the latter. This finding implies that therapeutic time window extends to $4.5 \mathrm{~h}$ after stroke onset.

Thus, clinical trials have shown that only t-PA is useful for the treatment of acute ischemic stroke. In this review, we focus on the mechanisms that explain the association between delayed t-PA treatment and the hemorrhagic transformation of ischemic stroke.

\section{2. t-PA induces rebleeding by binding to the hemo- static plug}

t-PA acts as a thrombolytic agent by degrading fibrin clots through the activation of plasminogen to plasmin (14). One of the reasons for the development of ICH after t-PA treatment is overdose. t-PA significantly prolongs the bleeding time and the duration of excessive rebleeding after the termination of t-PA infusion (15). t-PA treatment has also been associated with a tendency for bleeding persisting after the termination of t-PA infusion; this is believed to be caused by the binding of t-PA to the hemostatic plug. However, when administered in the effective dose, t-PA did not alter the activated partial thromboplastin time (aPTT), which shows that it does not affect the coagulation system.

\section{Reperfusion injury by t-PA treatment}

The other possible cause of ICH is reperfusion injury, which induces the release of free radicals. It is believed that free radicals could be involved in $\mathrm{ICH}$ associated with t-PA treatment. In clinical trials, disodium 2,4disulfophenyl- $N$-tert-butyl nitrone (NXY-059, a freeradical-trapping agent) showed promise as a neuroprotectant in the Stroke-Acute Ischemic NXY Treatment I (SAINT I) trial (16), reducing disability when given to patients who had acute ischemic stroke. Although the hypothesis that NXY-059 would reduce t-PA-related ICH was evaluated in SAINT II trial, it was similar between the patients showing the presence and those showing the absence of NXY-059 (4.6\% and 5.3\%, respectively) (17). However, edaravone, a radical scavenger, has been approved in Japan for use as a neuroprotectant in the treatment of acute ischemic stroke since 2001. 
According to a postmarketing surveillance of t-PA for stroke in Japan, the rate of occurrence of cerebral hemorrhage among patients treated with a combination of t-PA and edaravone could be lower than that among patients treated with t-PA only. The clinical trail of this combination therapy is currently underway in Japan, and the latest findings indicate that free radical formation is involved in the increased risk of ICH associated with this treatment.

\section{4. t-PA / plasmin / MMP pathway}

Another possible mechanism underlying the occurrence of hemorrhage due to t-PA treatment is the activation of matrix metalloproteinases (MMPs). Plasmin activates MMPs, a family of zinc endopeptidases, and thereby contributes to tissue remodeling through the degradation of extracellular matrix proteins (18). In the pathogenesis of ICH associated with ischemic stroke, MMPs play a key role in degrading the barrier of blood vessels $(19,20)$. We investigated the incidence of ICH induced by t-PA treatment in mouse models of thrombotic ischemic stroke with genetic deficiencies of plasminogen $\left(\mathrm{Plg}^{-/}\right)$, stromelysin-1 (MMP-3 $\left.3^{-/}\right)$, or gelatinase B (MMP-9 ${ }^{-/}$) and their corresponding wild-type (WT) littermates $\left(\mathrm{Plg}^{+/+},{\mathrm{MMP}-3^{+/+} \text {, and MMP-9 }}^{+/+}\right)(21)$. The incidence of t-PA-induced ICH was significantly lower in the $\mathrm{Plg}^{-/}$and MMP- $3^{---}$mice than in the corresponding WT mice, but this difference was not observed in the case of the MMP- $9^{-/-}$mice. A previous report indicated that MMP-9 expression is increased in endothelial cells (ECs) of the ischemic brain tissue after stroke (22), while our study reported that t-PA treatment does not alter either the amount or the distribution of MMP-9 in the brain (21). These findings suggest that MMP-9 may mediate the pathogenesis of ICH associated with ischemic stroke itself, rather than that induced by t-PA treatment. Thus, considering that the increase in the risk of ICH was not observed in MMP- $3^{-/-}$mice and that a broad-spectrum MMP inhibitor suppressed the increase in WT mice but not in MMP- $3^{-/-}$mice (21), it can be inferred that the increase in the risk of ICH by t-PA treatment may be attributable to MMP-3 induction. Since MMP-3 has broad substrate specificity for extracellular proteins (23), it plays a key role in tissue remodeling. Considering the abovementioned points, it is possible that MMP-3 induction by t-PA results in the acceleration of the cellular response upon remodeling.

\section{5. t-PA induces MMP-3 through the LRP / NF- $\kappa$ B pathway in endothelial cells}

MMP-3 expression was significantly enhanced in the ischemic brain tissue of mice, irrespective of whether or not t-PA was administered. However, the distribution of ischemia-induced MMP-3 in brain tissue varied with the type of affected cells; MMP-3 was induced in neurons but not in the ECs in the placebo-treated group, while it was significantly upregulated in the ECs in the t-PA treatment group (21). Furthermore, MMP-3 is induced by t-PA in bEnd. 3 cells, a mouse-derived cerebral endothelial cell line, under ischemic stress in vitro (24).

Recent studies have indicated that t-PA also acts through plasmin(ogen)-independent mechanisms, including low-density lipoprotein receptor-related protein (LRP) activation under various physiological and pathophysiological conditions $(22,25)$. LRP, a member of the lipoprotein receptor family, is a scavenger receptor that binds to a variety of biological ligands and is thought to be involved primarily in lipoprotein metabolism (26) and clearance of protease-inhibitor complexes in the adult brain (27) and, to some extent, in intracellular signal transduction (28). Furthermore, the coupling of the LRP and NF- $\kappa \mathrm{B}$ pathways has been observed in macrophages and ECs $(29,30)$. In addition, the MMP-3 induction associated with NF- $\kappa$ B activation has been widely observed in pathological conditions such as rheumatoid arthritis (31), although no canonical NF- $\kappa$ B sites were identified in the promoter sequence of MMP-3 (32). bEnd.3 cell experiments showed that MMP-3 expression was also induced by t-PA treatment and that it was suppressed either by the inhibition of LRP with receptor-associated protein (RAP), a general antagonist of members of the LDL receptor family; treatment with an anti-LRP antibody; or by inhibition of the NF- $\kappa \mathrm{B}$ pathway. These findings suggested that the induction of MMP-3 expression by t-PA also occurs via the LRP/NF- $\kappa$ B pathway in ECs (24). However, it has to be conceded that this pathway may also be induced by other pathways or proteins. Furthermore, pretreatment with RAP suppressed t-PAinduced MMP-3 expression at the border of the ischemiaaffected ECs in mice treated with t-PA (24), indicating that t-PA also induces MMP-3 via LRP in vivo.

\section{Effect of ischemia on LRP expression in endothelial cells}

When exposed to $6 \mathrm{~h}$ of ischemic stress, bEnd. 3 cells exhibited enhanced LRP production, both in terms of mRNA and protein levels. In addition, LRP expression in the ischemia-affected hemisphere was significantly upregulated at both 6 and $24 \mathrm{~h}$ of MCA occlusion in a mouse model of ischemic stroke, and this expression was mainly localized to globular CD31-positive ECs; no LRP expression was found in the unaffected hemisphere, similar to native mice. These in vitro and in vivo studies 
indicate that ischemic stress upregulates LRP expression in ECs several hours after the onset of ischemia. Since LRP functions as a receptor for t-PA, the sensitivity of endothelial cells to t-PA will be increased under ischemic conditions. This hypothesis is consistent with the clinical or experimental findings showing that the risk of $\mathrm{ICH}$ after stroke is not increased by early but by delayed treatment with t-PA $(11,15)$, even if t-PA is administered systemically. LRP upregulation was also observed in neurons under ischemic stress (33). Therefore, increased LRP expression in the ischemic hemisphere may be attributable to increased LPR expression not only in ECs but also neurons surrounding the damaged area.

\section{Therapeutic perspectives}

The widespread use of t-PA for acute ischemic stroke is hampered by 2 reasons: t-PA has proven therapeutically effective in only a narrow time window and it is associated with a high incidence of $\mathrm{ICH}$, which worsens the clinical outcome $(11,13)$. To explore the role of LRP in the t-PA-associated increase in the risk of $\mathrm{ICH}$, the effect of RAP was studied in a mouse model of ischemic stroke (24). Pretreatment with RAP led to a decrease in the risk of t-PA-induced $\mathrm{ICH}$, indicating that the induction of MMP-3 in ECs by t-PA via LRP may be associated with the risk of t-PA-induced ICH. In human ECs, t-PA also induced MMP-9 expression through its action on LRP (22). Clinically, suppression of both ICH induced by t-PA and that associated with ischemic stress itself would be beneficial. The increased risk of ICH caused by t-PA treatment was found to be impaired in mice with deficiency of the stromelysin-1 (MMP-3) gene, and treatment with some broad-spectrum MMP inhibitors was shown to reduce the risk of t-PA-induced ICH in rodent models $(19-21)$. Although human clinical trials on the amelioration of MMP-mediated brain damage have been unsuccessful because of low specificity and high toxicity of the tested therapeutic agents (34), highly selective MMP-3/MMP-9 inhibitors may be beneficial in this respect. The reduction of both the risk of ICH and t-PAinduced MMPs expression by RAP treatment suggests that t-PA-induced ICH can be suppressed by LRP inhibition. The increase in the risk of ICH and MMP-3 expression by t-PA treatment is mediated by the proteolytic activity of t-PA $(21,24)$, suggesting that receptor binding alone is not sufficient to trigger these effects. This finding is consistent with our previous findings indicating that plasminogen is essential for t-PA-mediated increase in the risk of $\mathrm{ICH}$ increase, thereby suggesting that plasmin generation is essential for t-PA to exert these effects $(21,24)$.

\section{Conclusions}

The risk of ICH associated with treatments in ischemic stroke is mainly attributable to antithrombotic agents. The widespread use of thrombolytic agents for the treatment of acute ischemic stroke is avoided because of its association with increased incidence of ICH, which worsens the patients' outcome. Although t-PA improves the outcome of ischemic stroke by promoting recanalization of the occluded brain vessels, it increases the risk of ICH when administered later than $3 \mathrm{~h}$ after the onset of ischemia. t-PA-induced ICH occurs via stimulation of MMP-3 expression in ECs, and this effect is mediated by the LRP / NF- $\kappa$ B pathway. Since only a small percentage of individuals affected by ischemic stroke reach the hospital in time to be considered for t-PA treatment, that is, within the 3-h therapeutic window from the onset of symptoms, strategies to increase the window period of the therapeutic effectiveness of t-PA would reduce the risk associated with this therapy and prove to be beneficial for patients with acute ischemic stroke. Induction of MMP-3 via the LRP / NF- $\kappa$ B pathway can be targeted to improve the therapeutic efficacy of t-PA for acute ischemic stroke.

\section{References}

1 Hankey G, Sudlow C, David W. Thienopyridines or aspirin to prevent stroke and other serious vascular events in patients at high risk of vascular disease? A systematic review of the evidence from randomized trials. Stroke. 2000;31:1779-1784.

2 Linkins L, Choi P, Douketis J. Clinical impact of bleeding in patients taking oral anticoagulant therapy for venous thromboembolism: a meta-analysis. Ann Intern Med. 2003;139:893-900.

3 European Atrial Fibrillation Trial (EAFT) Study Group. Secondary prevention in non-rheumatic atrial fibrillation after transient ischaemic attack or minor stroke. Lancet. 1993;342:1255-1262.

4 Cerebral Embolism Study Group. Immediate anticoagulation of embolic stroke: brain hemorrhage and management options. Stroke. 1984;15:779-789.

5 International Stroke Trial Collaborative Group. The International Stroke Trial (IST): a randomized trial of aspirin, subcutaneous heparin, both, or neither among 19435 patients with acute ischaemic stroke. Lancet. 1997;349:1569-1581.

6 Berge E, Abdelnoor M, Nakstad PH, Sandset PM. Low molecular-weight heparin versus aspirin in patients with acute ischaemic stroke and atrial fibrillation: a double-blind randomised study. HAEST Study Group. Heparin in Acute Embolic Stroke Trial. Lancet. 2000;355:1205-1210.

7 Shinohara Y, Katayama Y, Uchiyama S, Yamaguchi T, Handa S, Matsuoka K, et al. Cilostazol for prevention of secondary stroke (CSPS 2): an aspirin-controlled, double-blind, randomised noninferiority trial. Lancet Neurol. 2010;9:959-968.

8 Fletcher A, Alkjaersig N, Lewis M, Tulevski V, Davies A, Brooks JE, et al. A pilot study of urokinase therapy in cerebral infarction. Stroke. 1976;7:135-142. 
9 Furlan A, Higashida R, Wechsler L, Gent M, Rowley H, Kase C, et al. Intra-arterial prourokinase for acute ischemic stroke. The PROACT II study: a randomized controlled trial. Prolyse in Acute Cerebral Thromboembolism. JAMA. 1999;282:20032011.

10 Ogawa A, Mori E, Minematsu K, Taki W, Takahashi A, Nemoto S, et al. MELT Japan Study Group. Randomized trial of intraarterial infusion of urokinase within 6 hours of middle cerebral artery stroke: the middle cerebral artery embolism local fibrinolytic intervention trial (MELT) Japan. Stroke. 2007;38:2633-2639.

11 Hacke W, Donnan G, Fieschi C, Kaste M, von Kummer R, Broderick J, et al. Association of outcome with early stroke treatment: pooled analysis of ATLANTIS, ECASS, and NINDS rt-PA stroke trials. Lancet. 2004;363:768-774.

12 Wahlgren N, Ahmed N, Davalos A, Ford GA, Grond M, Hacke W, et al. Thrombolysis with alteplase for acute ischaemic stroke in the safe implementation of thrombolysis in stroke-monitoring study (SITS-MOST): an observational study. Lancet. 2007;369: 275-282.

13 Bluhmki E, Chamorro A, Dávalos A, Machnig T, Sauce C, Wahlgren N, et al. Stroke treatment with alteplase given 3.0-4.5 h after onset of acute ischaemic stroke (ECASS III): additional outcomes and subgroup analysis of a randomised controlled trial. Lancet Neurol. 2009;8:1095-1102.

14 Lijnen H, Collen D. Tissue-type plasminogen activator. Ann Biol Clin (Paris). 1987;45:198-201.

15 Suzuki Y, Nagai N, Collen D. Comparative effects of microplasmin and tissue-type plasminogen activator (tPA) on cerebral hemorrhage in a middle cerebral artery occlusion model in mice. J Thromb Haemost. 2004;2:1617-1621.

16 Lees KR, Zivin JA, Ashwood T, Davalos A, Davis SM, Diener $\mathrm{HC}$, et al. NXY-059 for acute ischemic stroke. N Engl J Med. 2006;354:588-600.

17 Shuaib A, Lees K, Lyden P, Grotta J, Davalos A, Davis S, et al. NXY-059 for the treatment of acute ischemic stroke. N Engl J Med. 2007;357:562-571.

18 Lijnen H. Plasmin and matrix metalloproteinases in vascular remodeling. Thromb Haemost. 2001;86:324-333.

19 Lapchak P, Chapman D, Zivin J. Metalloproteinase inhibition reduces thrombolytic (tissue plasminogen activator)-induced hemorrhage after thromboembolic stroke. Stroke. 2000;31: 3034-3040.

20 Sumii T, Lo E. Involvement of matrix metalloproteinase in thrombolysis-associated hemorrhagic transformation after embolic focal ischemia in rats. Stroke. 2002;33:831-836.

21 Suzuki Y, Nagai N, Umemura K, Collen D, Lijnen $H$. Stromelysin-1 (MMP-3) is critical for intracranial bleeding after t-PA treatment of stroke in mice. J Thromb Haemost. 2007;5:
$1732-1739$.

22 Wang X, Lee S, Arai K, Lee S, Tsuji K, Rebeck G, et al. Lipoprotein receptor-mediated induction of matrix metalloproteinase by tissue plasminogen activator. Nat Med. 2003;9:1313-1317.

23 Nagase H, Woessner J Jr. Matrix metalloproteinases. J Biol Chem. 1999;274:21491-21494.

24 Suzuki Y, Nagai N, Yamakawa K, Kawakami J, Umemura K. Tissue-type plasminogen activator (t-PA) induces stromelysin-1 (MMP-3) in endothelial cells through activation of lipoprotein receptor-related protein. Blood. 2009;114:3352-3358.

$25 \mathrm{Hu} \mathrm{K}$, Wu C, Mars W, Liu Y. Tissue-type plasminogen activator promotes murine myofibroblast activation through LDL receptorrelated protein 1-mediated integrin signaling. J Clin Invest. 2007; 117:3821-3832.

26 Herz J, Hamann U, Rogne S, Myklebost O, Gausepohl H, Stanley K. Surface location and high affinity for calcium of a 500-kd liver membrane protein closely related to the LDL-receptor suggest a physiological role as lipoprotein receptor. EMBO J. 1988; 7:4119-4127.

$27 \mathrm{Bu} \mathrm{G}$, Williams S, Strickland D, Schwartz A. Low density lipoprotein receptor-related protein/alpha 2-macroglobulin receptor is an hepatic receptor for tissue-type plasminogen activator. Proc Natl Acad Sci U S A. 1992;89:7427-7431.

28 Lillis A, Van Duyn L, Murphy-Ullrich J, Strickland D. LDL receptor-related protein 1: unique tissue-specific functions revealed by selective gene knockout studies. Physiol Rev. 2008;88:887918.

29 Rigau V, Morin M, Rousset M, Bock F, Lebrun A, Coubes P, et al. Angiogenesis is associated with blood-brain barrier permeability in temporal lobe epilepsy. Brain. 2007;130:1942-1956.

30 Cheng S, Xing B, Li J, Cheung B, Lau A. Interferon-gamma regulation of TNFalpha-induced matrix metalloproteinase 3 expression and migration of human glioma T98G cells. Int J Cancer. 2007;121:1190-1196.

31 Bondeson J, Foxwell B, Brennan F, Feldmann M. Defining therapeutic targets by using adenovirus: blocking NF-kappaB inhibits both inflammatory and destructive mechanisms in rheumatoid synovium but spares anti-inflammatory mediators. Proc Natl Acad Sci U S A. 1999;96:5668-5673.

32 Westermarck J, Kahari VM. Regulation of matrix metalloproteinase expression in tumor invasion. FASEB J. 1999;13:781-792.

33 Zhang X, Polavarapu R, She H, Mao Z, Yepes M. Tissue-type plasminogen activator and the low-density lipoprotein receptorrelated protein mediate cerebral ischemia-induced nuclear factorkappaB pathway activation. Am J Pathol. 2007;171:1281-1290.

34 Coussens L, Fingleton B, Matrisian L. Matrix metalloproteinase inhibitors and cancer: trials and tribulations. Science. 2002;295: 2387-2392. 